\title{
Typhoid fever, ciprofloxacin, and renal failure
}

\author{
J Simpson, A R Watson, A Mellersh, C S Nelson, K Dodd
}

\begin{abstract}
An 11 year old boy from whom Salmonella typhi had been isolated was treated with ciprofloxacin. He developed non-oliguric acute renal failure that was treated successfully.

Ciprofloxacin has recently been suggested as the preferred treatment for traveller's diarrhoea and multiresistant Salmonella typhi infection. ${ }^{1}$ The latter problem is being seen increasingly often in the United Kingdom. ${ }^{2}$ Although the drug is not recommended for children, it is an accepted oral treatment for pseudomonal infections, particularly infective exacerbations of cystic fibrosis. ${ }^{3}$ We report a case of non-oliguric acute renal failure caused by interstitial nephritis that developed after treatment of typhoid fever with ciprofloxacin.
\end{abstract}

\section{Case report}

An 11 year old boy was admitted with a four day history of pyrexia, rigors, diarrhoea, and vomiting. He had returned 10 days earlier from a month's holiday in Pakistan. He had received no immunisation against typhoid or cholera and no prophylaxis against malaria. The $S$ typhi phage type $\mathrm{Ml}$ that was isolated from his blood and stools was resistant to ampicillin, trimethoprim, and chloramphenicol but sensitive to cefuroxime, gentamicin, and ciprofloxacin. The boy was $152 \mathrm{~cm}$ tall and weighed $32.7 \mathrm{~kg}$.

Chloramphenicol was prescribed for one day, gentamicin for two days $(7 \cdot 3 \mathrm{mg} / \mathrm{kg}$ body weight/day), and cefuroxime $(90 \mathrm{mg} / \mathrm{kg}$ body weight/day) for seven days. Three days after starting cefuroxime his fever had not settled and ciprofloxacin was added as this antibiotic was felt to be more bactericidal in a resistant $S$ typhi infection. Two initial doses of oral ciprofloxacin were followed by six days of intravenous treatment, the dose being $150 \mathrm{mg}$ twice a day $(9 \mathrm{mg} /$ $\mathrm{kg}$ body weight/day) with a further two days of oral treatment ( $15 \mathrm{mg} / \mathrm{kg}$ body weight/day). The child's fever settled within 36 hours of starting ciprofloxacin.

The plasma urea and creatinine concentrations before treatment were $3.7 \mathrm{mmol} / \mathrm{l}$ and 74 $\mu \mathrm{mol} / \mathrm{l}$, respectively. Analysis of urine on admission showed neither blood nor protein. The patient initially responded well, but two weeks later he developed lethargy and anorexia with occasional vomiting. The urea and creatinine concentrations had risen to $37 \mathrm{mmol} / \mathrm{l}$ and $1183 \mu \mathrm{mol} / \mathrm{l}$, respectively. He continued to pass urine, which now showed moderate glycosuria, haematuria, and proteinuria, with no casts or

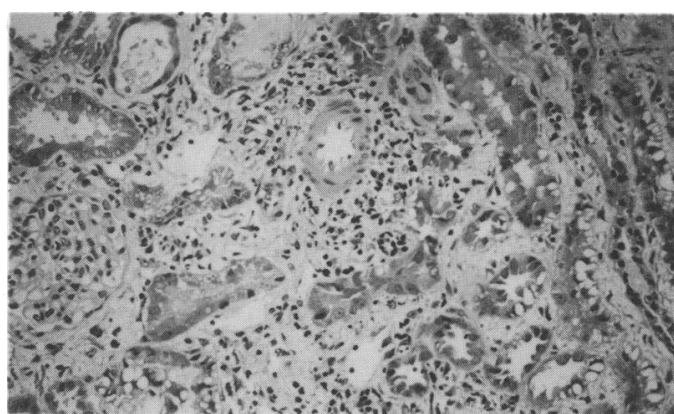

Figure 1 Photomicrograph of a renal biospy specimen at a magnification of 260 showing interstitial nephritis with pronounced oedema and mixed infiltrate of chronic inflammatory cells.

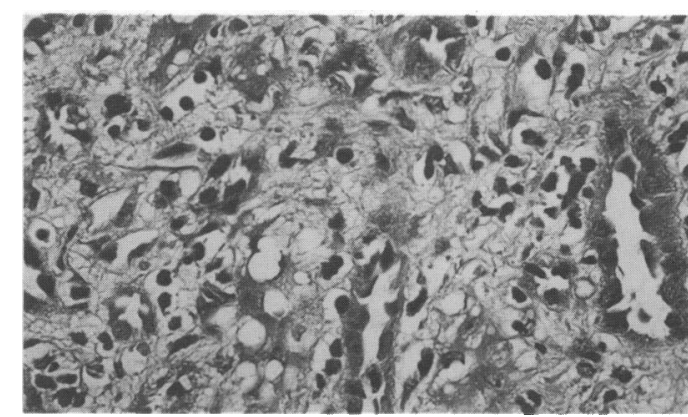

Figure 2 Photomicrograph of a renal biopsy specimen at a magnification of 650 showing acute interstitial nephritis with a mixed chronic inflammatory cell infiltrate and invasion of tubules by lymphoid cells.

crystals. The blood film did not show any eosinophilia, the haemoglobin concentration was 87 $\mathrm{g} / \mathrm{l}$, and the white cell count $6.3 \times 10^{9} / 1$. Ciprofloxacin was discontinued, but after a week of further observation and conservative management his plasma creatinine concentration remained at $1133 \mu \mathrm{mol} / \mathrm{l}$.

A percutaneous renal biopsy was done, which showed clear evidence of interstitial nephritis (figures 1 and 2). There was no evidence of immunoglobulin or complement deposition on immunofluorescent staining and electron microscopy of the glomeruli showed no abnormality. Prednisolone $70 \mathrm{mg}$ daily $(2 \cdot 1 \mathrm{mg} / \mathrm{kg}$ body weight/day) was given orally for three days and tapered off over a week when the plasma creatinine concentration had fallen to $155 \mu \mathrm{mol} / \mathrm{l}$. There was no recurrence of the typhoid fever and two weeks later the creatinine concentration had fallen to $79 \mu \mathrm{mol} / \mathrm{l}$.

\section{Discussion}

The clinical course in this case is consistent with ciprofloxacin induced interstitial nephritis 
causing non-oliguric renal failure. This has been described in a few case reports. ${ }^{4-6}$ In only one patient was the diagnosis proved on histological examination, ${ }^{4}$ and there have been no cases reported in children to our knowledge. Although our patient also received cefuroxime we are unaware of any cases of interstitial nephritis induced by cefuroxime given either alone or with other drugs.

Ciprofloxacin is one of a relatively new group of antimicrobial agents, the quinolones, the indications for which are increasing. Many side effects have been reported and the drug, like others in its class, is not recommended for children and adolescents because of the reported arthropathy in weight bearing joints of immature animals. Range of activity of ciprofloxacin and availability in an oral form has made it an attractive choice for the treatment of pseudomonal and other infections, particularly in patients with cystic fibrosis and urological problems.

All patients with ciprofloxacin associated renal failure described by the time of writing have maintained a urine output. Detection therefore depends upon biochemical tests. We suggest that renal function should be monitored during treatment with ciprofloxacin.. In addition, ciprofloxacin should be added to the long list of drugs that may cause interstitial nephritis.

We thank Dr S Boyd for providing the photomicrographs.

1 Anonymous. Quinolones in acute non-travellers' diarrhoea [Editorial]. Lancet 1990;336:282-3.

2 Rowe B, Ward LR, Threlfull EJ. Spread of multiresistant Salmonella typhi. Lancet 1990;336:1065.

3 Scully BE, Nakatomi M, Ores C, Davidson S, Neu HC. Ciprofloxacin therapy in cystic fibrosis. Am $\mathcal{f}$ Med 1987;8 (suppl 4A): 196-201.

4 Rippelmeyer DJ, Synhavasky A. Ciprofloxacin and allergic interstitial nephritis. Ann Intern Med 1988;109:170.

5 Hootkins R, Fenves AZ, Stephens MK. Acute renal failure secondary to oral ciprofloxacin therapy: a presentation of three cases and a review of the literature. Clin Nephrol 1989;32:75-8.

6 Ying LS, Johnson CA. Ciprofloxacin-induced interstitial nephritis. Am $\mathcal{F}$ Hosp Pharm 1989;46:1658.

\section{Down's syndrome}

The April issue of Developmental Medicine and Child Neurology contains three articles about Down's syndrome. In the first of these, Cliff Cunningham and his coworkers (pages 285-95) report on 123 children and their families in Manchester addressing the question of whether development and social adaptation are related to appearance. Checklists completed by teachers were used to assess appearance and 'attractiveness' and child and family functioning were measured by psychologists and from data obtained from parents by questionnaire and interview. The authors conclude that physical appearance is not a major determinant of development or social functioning in Down's syndrome and that their findings would not support attempts to alter the appearance by surgery. A possible drawback of the study is that the assessments of appearance and attractiveness were done by teachers and therefore might not be devoid of bias vis-a-vis the viewpoint of the general population.

The second paper is from Munich (GJ Limbrock and colleagues, pages 296-303) and describes the use of Castillo-Morales' orofacial therapy in which a 'manual stimulation and facilitation programme' is combined with the use of an oral plate designed to stimulate the palate, tongue, and lips. I suspect that this is the kind of treatment which needs to be seen in action in order to get to grips with it. I found that the results were presented in a way which was difficult to assess but 67 children were treated and the authors claimed significant improvements in tongue position, lip tone, mouth closure, drooling, and sucking. Unfortunately the study was uncontrolled.

The third paper is about the attitudes of American doctors to the treatment of duodenal atresia in Down's syndrome (Mark L Wolraich and colleagues, pages 336-42). They surveyed a sample of paediatricians, paediatric surgeons, and general practitioners and found that more would recommend surgery than has been the case in the fairly recent past. Perhaps not surprisingly the surgeons tended to be the least optimistic about prognosis and the least likely to recommend surgery. 